\title{
An Approach to Service and Cloud Computing Oriented Web GIS Application
}

\author{
Xiaolin $\mathrm{Lu}$ \\ College of Information and Electronic Engineering \\ Zhejiang University of Science and Technology \\ Hangzhou, China, 310012 \\ xiaolinlu@china.com.cn
}

\begin{abstract}
The service and cloud computing oriented architecture is a very promising architecture for practical implementation of the next generation geographical information systems. This paper investigated the service and cloud computing oriented architecture for constructing a distributed and web service enabled geographical information platform. The spatial cloud computing applies geographical information systems to the cloud computing paradigm. It provides dynamically scalable geographic information technology, spatial data, and spatial applications as a web service. GIS web services were designed to provide the hosted spatial data and GIS functionality to integrate the customized GIS applications to perform basic geo-processing tasks, such as address matching, map image display, and routing, without maintaining GIS tools or the associated geographical data. The system architecture, functions, system integrations, and some key technical problems are investigated. It has an important application prospect in the GIS tools development and application.
\end{abstract}

\section{Keywords-GIS; J2EE; Web Service; Cloud Computing}

\section{INTRODUCTION}

Cloud Computing is an approach to computing in which dynamically scalable computing hardware and software resources are provided as a service over the Internet [1-3]. With cloud computing, the cloud computing oriented applications presents on-demand self-service, ubiquitous network access, location-independent resource pooling, rapid elasticity, and pay-per-use. It has three delivery models, SaaS (software as a service), PaaS(platform as a service), IaaS(infrastructure as a service)[4,5]. The Google's Google Reader and Google Docs is the SaaS examples for cloud computing [6]. Amazon's Elastic Compute Cloud provides compute capacity in the cloud and is a leading IaaS. Cloud Computing offers cost effective solutions that are dependable, and reliable. The value of the Cloud Computing industry is expected to reach hundreds Billion in five years.

Spatial Cloud Computing researches to apply geography to the cloud computing paradigm [6, 7]. It provides dynamically scalable geographic information technology, spatial data, and spatial applications as a web service. The applications do not need the massive investment in specialized spatial data, and software typically required in an enterprise GIS implementation. It has the benefits of all the cloud computing.
With spatial cloud computing, The Spatial Cloud Computing provides the foundation data as part of the core service.

Web Services are the software components that can be accessed through the Internet in other applications. GIS Web services are the software components that can provide the hosted spatial data and GIS functionalities and can be accessed and integrated to the practical customized GIS applications. Developers can apply the GIS Web services to perform geographical information processing and return the results to the customized applications without maintaining the basic GIS system or the geographical data. The service and cloud computing oriented architecture is a very promising architecture for practical implementation of the next generation geographical information systems.

The GIS web services have been thoroughly investigated in recent years [8]. There are a number of researches and developing projects of applying the web services to GIS system by several different companies and organizations. The Open GIS Consortium (OGC) developed the web-based geographic information and services accessible via the Internet through the OGC consensus process [9]. Each service supports a certain GIS operation function that returns metadata describing the nature of the service, the methods to register Web Service, the addresses to access the service or to contact its provider, the type of data provided by the service, and so forth. The Environmental Systems Research Institute (ESRI) reported the ArcWeb Services that could include and integrate the GIS content and capabilities in the customized applications for sharing geographical information and data [9]. Microsoft MapPoint .NET is a hosted, programmable XML Web Service that allows application developer to integrate high-quality maps, driving directions, distance calculations, and proximity searches into their applications, business processes, and web sites.

Although many researches have been devoted to apply the web services technology to GIS system with different kinds of GIS Web Services, few studies have been reported on the architecture design for building the Web service enabled GIS system platform. The purpose of this paper is to explore and investigate the service and cloud computing oriented architecture for building a distributed and web service enabled GIS platform. The research aims to apply the web service framework into the GIS system design and implementation. 
This paper investigated the service oriented system architecture for constructing the distributed Web GIS platform and its practical application. The advantages of the architecture, functions, system integrations, and some key technical problems were discussed in this paper.

\section{OVERVIEW OF RELATED WORKS}

\section{A. Cloud Computing}

Cloud computing is a way of computing shares computer resources on Internet instead of using software or storage on a local computer [1-3]. Cloud computing is an outgrowth of the ease-of-access to remote computing sites provided by the Internet. In concept, it is a paradigm shift whereby details are abstracted from the users who no longer have need of, expertise in, or control over the technology infrastructure in the cloud that supports them. Cloud computing describes a new supplement, consumption and delivery model for IT services based on the Internet, and it typically involves the provision of dynamically scalable and often virtualized resources as a service over the Internet. The term cloud is used as a metaphor for the Internet, based on the cloud drawing used to depict the Internet in computer network diagrams as an abstraction of the underlying infrastructure it represents. Typical cloud computing providers deliver common business applications online which are accessed from a web browser, while the software and data are stored on servers.

A technical definition is a computing capability that provides an abstraction between the computing resource and its underlying technical architecture, enabling convenient, ondemand network access to a shared pool of configurable computing resources that can be rapidly provisioned and released with minimal management effort or service provider interaction. This definition states that clouds have five essential characteristics: on-demand self-service, broad network access, resource pooling, rapid elasticity, and measured service. Narrowly speaking, cloud computing is client-server computing that abstract the details of the server away - one requests a service, not a specific server.

The majority of cloud computing infrastructure consists of reliable services delivered through data centers and built on servers. Clouds often appear as single points of access for all consumers' computing needs.

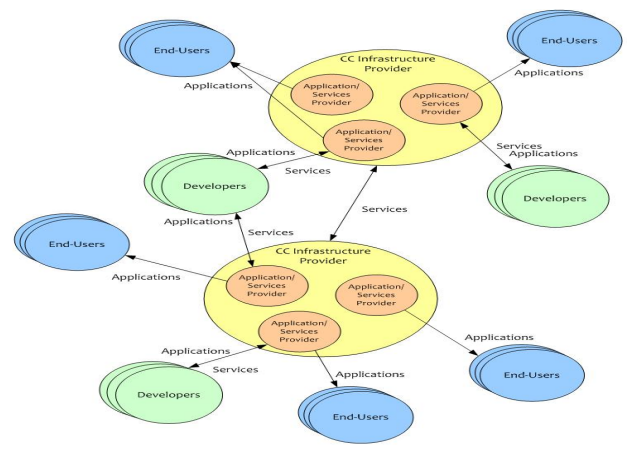

Figure 1. Cloud Computing Architecture

\section{B. $\quad$ Services oriented architecture}

Web Services are the software components that are well defined, self-contained, and does not depend on the context or state of other services [10]. Web services essentially use XML to create a robust connection. The web services architecture has three roles: a provider, a requestor, and a broker. The provider creates the web service and makes it available to clients who want to use it. A requestor is a client application that consumes the web service. The broker, such as a service registry, provides a way for the provider and the requestor of a web service to interact. The provider, requestor, and broker interact with each other through the operations of publish, find, and bind. A provider informs the broker about the existence of the web service by using the broker's publish interface to make the service accessible to clients. The information published describes the service and specifies where the service is located. The requestor consults the broker to locate a published web service. With the information it gained from the broker about the web service, the requestor is able to bind, or invoke, the web service. The following figure illustrates a basic service and cloud computing oriented architecture.

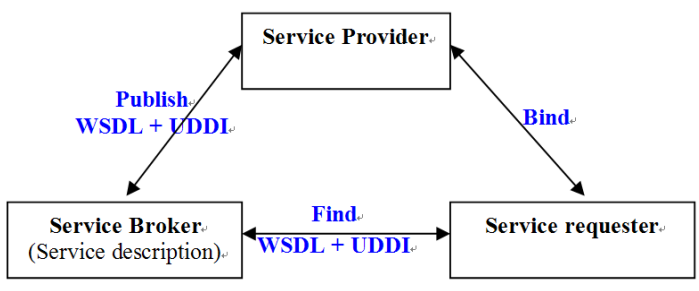

Figure 2. Web services oriented architecture

The web services use SOAP (Simple Object Access Protocol), WSDL (Web Services Description Language), UDDI (Universal Description, Discovery and Integration), and WSIL (Web Services Inspection Language) to communicate. Web services are published on the UDDI registry. A WSDL document defines the target service so the client knows what the service does. SOAP standardizes the way a Web service communicates with a client and allows programs written in different languages and on different platforms to communicate. SOAP works with standard Web protocols including XML, HTTP, and TCP/IP, as well as WSDL.

\section{Open GIS Consortium Web Services (OWS)}

OGC is an international industry consortium of more than 220 companies, government agencies and universities. In order to allow interoperability between server and client software from multiple vendors, the request and response must be standardized. By focusing standardization on the interface, the software developers, data custodians, and processing-service providers can work to their particular software components, while connecting with other systems for complementary services. Open GIS Consortium is developing specifications to standardize the message interfaces [9]. These include

Web Map Service (WMS) for maps encoded as images;

Web Feature Service (WFS) for geographic objects or vector data; 
Web Coverage Service (WCS) for continuous data;

Sensor Collection Service (SCS) for live access to observations from sensors;

Geography Markup Language (GML): XML components for encoding geographic objects for transport.

\section{OGC Web Map Service (WMS)}

The OGC WMS specification offers a standard clientserver interaction protocol that each map server implements as a common interface for accepting requests and returning responses [8]. The same client is able to access to all available OGC web map servers over the Internet. The client through the common interface accesses each map server. In a distributed OGC WMS, a WMS server can also run as a WMS client that requests capabilities and maps from other WMS servers.

An OGC web map server implements three functions: GetCapabilities, GetMap, and GetFeatureInfo. The GetCapabilities function provides the client with a map server's service metadata, specifying its capabilities. The GetMap function specifies map request parameters that enable the client to request an image map. Finally, the GetFeatureInfo function allows the client to request more information about features at a specific location in the map.

A system structure of WMS mapping application is illustrated in Figure 3.

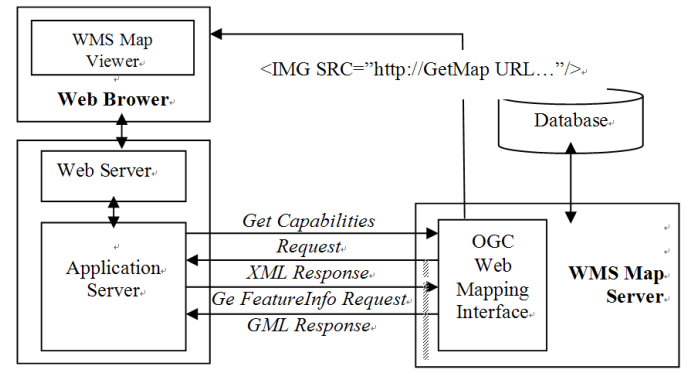

Figure 3. The system architecture of WMS mapping application

The client application requests the information from the web map server. The map server retrieves from the database the appropriate layers of geo-feature data for the specified spatial domain and generates a map that can be viewed directly in a graphical web browser or other pictorial software. The client and web map server interact using Hypertext Transfer Protocol.

OGC WMS is an interoperable web mapping system. It provides common interfaces to connect with the client application and dynamically process geo-referenced data such as geographic maps and features coded using Geography Markup Language (GML) documents. The OGC Web Map Service specification defines a set of functions that clients may use to interact with WMS providers (servers). Any client making requests that conform to the specification can interact with any server that implements the WMS service. In effect, this creates an interoperable, distributed web mapping system.

\section{SERVICE ORIENTED ARCHITECTURE DESIGN FOR DISTRIBUTED GIS PLATFORM}

The goal of the Service Oriented Distributed GIS platform is to provide both the dynamic GIS service components for publication vector and raster maps to Internet and interactive viewing on web browsers. It supports the complex rendering, navigation and allows working with multiple layers, thematic maps, hyper linked features and attribute data. The fundamental GIS platform is based on the Client/Server architecture. The server spreads vector maps to client. The map features are stored on a SQL database according OGC SFS and WMS that can be used as raster maps source. The GIS data interface supports the ArcView shape files and MapInfo MIF files. The server side is constructed based on J2EE architecture.

GIS Web Services are discoverable, self-describing software components. UDDI is an open standard with broad industry support standard. When an implemented GIS Web Service exposed in any Web Services portal, it can be discovered in any Web Services portal. Once a GIS Web Service is discovered, the developer can begin using it immediately. All they need is the full URL path to the services WSDL. Each method, parameter, property, and return value of the service is described in a standard way, allowing modern development tools to immediately allow access to the exposed functionality.

GIS Web Services conceal complexity. Web Services embed its complex data processing within itself in server side. Client side application developer make use of Web Services through standard interface which described in Web Services WSDL, therefore the application developer don't need dealing with Web Services internal process.

The following principles are considered in the design for the service oriented distributed GIS platform. Web GIS platform should provide a variety of interfaces for integration and interoperability with business and GIS applications.

GIS Web services: Web GIS platform should support the standard enterprise application server technologies to build web services with embedded spatial services, software components and APIs to easily embed spatial capabilities in business applications built using standard technologies and architectures.

Web Mapping Services (WMS): WMS can display map layers from other GIS applications, and allow other applications to display map layers from Web GIS platform

OpenGIS Consortium interoperability services and standards: The Web GIS platform should be implemented with the OpenGIS interoperability standards:

Geography Markup Language (GML): GML is a common XML-based file format for exchanging spatial information and attributes. The Web GIS platform should support the GML standards.

Common database environment: Specifications on spatial entities and the space/time reference systems have been provided by the OpenGIS Consortium. 


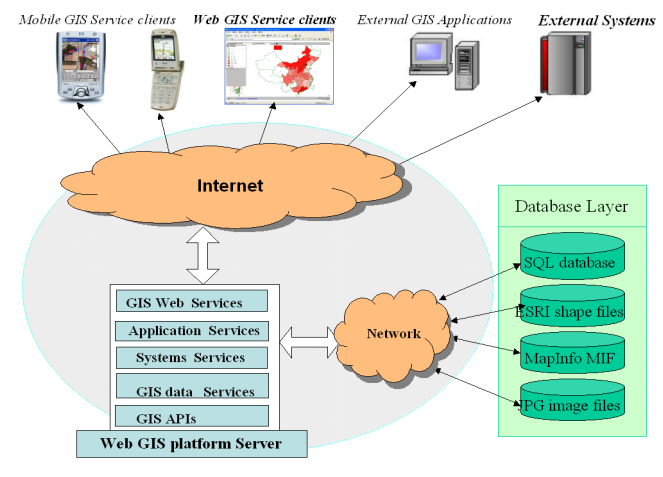

Figure 4. Web GIS platform server environment

\section{IMPLEMENTATION AND FURTHER WORK}

According to the service and cloud computing oriented architecture proposed in above section, we designed and implemented a prototype of the service enabled GIS platform. The GIS platform architecture is designed as a multi-layer architecture that integrated the web service, Servlet/JSP functions and GIS APIs based on the framework of J2EE infrastructure. It is a distributed, platform independent system architecture that be accessed by lots of different computers in network with different kinds of operating system.

The web service framework was applied into the GIS system design. GIS web services can provide hosted spatial data and GIS functionality and integrate to the customized GIS applications to perform basic geo-processing tasks, such as address matching, map image display, and routing, without maintaining GIS tools or the associated geographical data.

We have applied and developed the GIS platform to build up SARS prevention GIS application system. GIS Web service was used to get and show the geographical information. The application integrated the GIS Services and SARS information Service into a java applet in browser to reporting the most recent SARS case information.

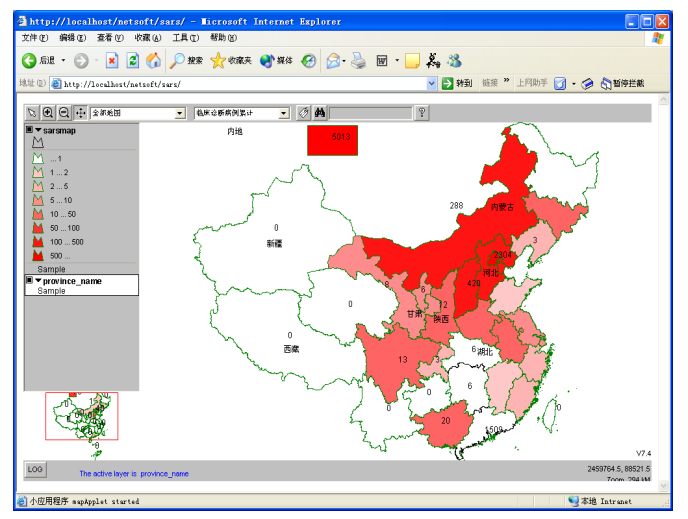

Figure 5. A screenshot of SARS prevention GIS application system.

The SARS GIS system provided a visual work environment for public and hygiene department to research, statistical analyze and broadcast the information of SARS epidemic situation according to the visual relationship between the spatial graphical data and the virus. The screenshot of the system is shown in figure 5.

\section{CONCLUSIONS}

This paper presented a service and cloud computing oriented architecture for constructing a distributed and web service enabled geographical information platform. The GIS platform architecture is designed as a multi-layer architecture that integrated the web service, Servlet/JSP functions and GIS APIs based on the framework of J2EE infrastructure. It is a distributed, platform independent system architecture that be accessed by lots of different computers in network with different kinds of operating system. The web service framework was applied into the GIS system architecture design. We designed and implemented a prototype of the service enabled GIS platform and presented an practical application of the SARS prevention GIS system. The GIS Web service was used to get and show the geographical information and integrated with the SARS information service into a java applet in browser to reporting the most recent SARS information. The service and cloud computing oriented architecture is a very promising architecture for practical implementation of the next generation geographical information systems. It could have an important application prospect in the GIS tools development and application.

\section{REFERENCES}

[1] B. Hayes, "Cloud computing," 2008.

[2] A. Weiss, "Computing in the clouds," COMPUTING, vol. 16, 2007.

[3] D. Nurmi, et al., "The eucalyptus open-source cloud-computing system," 2009, pp. 124-131.

[4] G. Zhou and G. He, "One Program Model for Cloud Computing," Cloud Computing, pp. 589-594.

[5] M. Vouk, "Cloud computing CIssues, research and implementations," Journal of Computing and Information Technology, vol. 16, pp. 235246, 2008.

[6] D. Sui, "GIS, cartography, and the third culture: Geographic imaginations in the computer age," The Professional Geographer, vol. 56, pp. 62-72, 2004.

[7] Shengru Tu, Maik Flanagin, Ying Wu, Mahdi Abdelguerfi, Eric Normand, Venkata Mahadevan, Jay J. Ratcliff and Kevin Shaw, "Design Strategies to Improve Performance of GIS Web Services", International Conference on Information Technology, Volume 2, 2004, pp. 444 449.

[8] Jong Woo Kim, Seong-Seok Park, Chang-Soo Kim and Yugyung Lee, "The Efficient Web-Based Mobile GIS Service System through Reduction of Digital Map", Lecture Notes in Computer Science 3043, Springer, 2004, pp. 410 417.

[9] Jihong Guan, Leichun Wang, and Shuigeng Zhou, "Enabling GIS Services in a P2P Environment" , 2004 International Conference on Computer and Information Technology, 2004, pp. 776 781.

[10] Ming-Hsiang Tsou, "An Operational Metadata Framework for Searching, Indexing, and Retrieving Distributed Geographic Information Services on the Internet", Lecture Notes in Computer Science 2478, Springer, 2002, pp. 313 332. 